\title{
On the Influence Venture Capital Exerts on SMEs Technology Innovation
}

\author{
Zhiwu Chen* and Ying Chen \\ School of Business, Hunan University of Technology, Zhuzhou Hunan, China \\ *Corresponding author
}

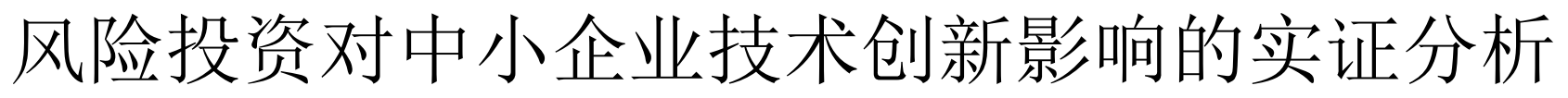

\author{
陈支武"，陈颖 \\ 湖南工业大学商学院, 株洲,中国 \\ 通讯作者
}

\begin{abstract}
Based on the GEM Board panel data of 287 listed companies in the manufacturing industry. A regression model and a negative binomial distribution model with venture capital as explaining variable and technology innovation as explained variable were built by the researcher. This paper makes an empirical analysis of influence that venture capital participation and participation degree exert on SMEs technology innovation input and output. Research and analysis showed that the participation of venture capital plays positive role in promoting small and medium enterprises technology innovation input and output; But it is not true to the higher shareholding ratio, the more output. There will be a negative effect if the shareholding ratio beyond certain limit. The participation degree of Venture capital has no significant influence. So venture capital should be introduced to the SMEs technology innovation activities but it ratio should not exceed certain limit.
\end{abstract}

Keywords-technology innovation; SMEs, venture capital; innovation input; innovation output

摘要一文章基于创业板 287 家制造业上市公司面板数据, 先 后构建了以风险投资为解释变量、以技术创新为被解释变量的回 归模型与负二项模型。对风险投资参与与持股比例对企业技术创 新投入与产出所带来的影响进行了实证分析。结果显示, 风险 投资参与对被投资企业的创新资源投入与产出均有强力促进作 用; 风险投资持股比例对技术创新的产出有促进作用, 但是超 过一定限度, 就会有反向影响; 而风险投资持股比例对技术创 新投入没有显著的影响。因此中小企业技术创新活动引入风险 投资, 对于提高其创新绩效具有积极意义, 但持股比例应控制 在一定范围内。

关键词一风险投资; 中小企业; 技术创新; 创新投入; 创新 产出

\section{I. 引言}

当前研究认为风险投资适应了企业技术创新的高风险、 高投入、高效益的特点, 二者具有天然的资源互补性, 是 企业, 特别是中小企业技术创新的主要融资渠道 ${ }^{[1-4]}$ 。同时 风险投资作为一种权益资本, 通过参与企业管理、监督企 业资源使用、为企业提供诸如营销、财务等增值服务能使 企业技术创新效率和创新能力明显提升 ${ }^{[5-6]}$ 。这些研究主要 关注“风险投资”作为一个整体对企业技术创新“能力”这一 要素的影响。本文将“风险投资”这一整体分解为“参与与与 “持股比例”两个要素, 而将“技术创新”分解为“技术创新投 入”与“技术创新产出”两个维度, 通过构建中小企业面板数 据模型，以深入考察风险投资对企业技术创新所带来的影 响。

\section{II. 理论分析与研究假设}

\section{A. 风险投资参与对企业技术创新的影响}

“拥有丰富经验的风险投资家对企业运营的高度参与可 以提高企业在创业和发展过程中各种项目的成功率”[7] (Keuschning，2004）。刘良（2013）、徐欣（2015）等 通过实证研究认为风险投资对企业技术创新具有显著的正 向促进作用 ${ }^{[8-11]}$ 。为此, 本文提出: 响。

假设 1: 风险投资参与对企业的技术创新投入有正向影 假设 2 : 风险投资参与对企业的技术创新产出有正向影

\section{B. 风险投资持股比例对企业技术创新的影响}

风险投资的逐利性激励着其对高新技术开发领域投资 的积极性, 并促使其动员企业将更多的资源用于研发新技 术、新产品。而王玉荣（2009）、赵武（2015）等的实证 研究结果也认为, 风险投资持股比例越高, 企业的技术创 
新投入越积极, 并且技术创新产出的能力也越强 ${ }^{[12-14]}$ 。由 此, 本文提出:

假设 3: 风险投资持股比例越高, 被投资企业技术创新 投入强度越大。

假设 4: 风险投资持股比例越高, 被投资企业技术创新 产出水平越高。

\section{III. 变量设计、样本选取与数据来源}

A. 变量设计

本文将主要的解释变量设为“风险投资”, 而将被解释变 量设为“技术创新”，同时还将其他可能影响企业技术创新 活动的各因素设为控制变量。具体解释如下:

(1) 解释变量。本文将解释变量“风险投资”分解为“有 无风险投资背景”和“风险投资持股比例”两个子变量。有则
为 1 , 无则为 0 。风险投资持股比例以企业招股说明书上企 业发行前的前十大股东可中风险投资机构持股比例之和确 定。

（2）被解释变量。被解释变量“技术创新”分解为“技术 创新投入”与“技术创新产出”两个子变量。实证分析中, 又 将 $R \& D$ 经费投入和 $R \& D$ 人员投入设为两个独立指标引入 模型来测度技术创新的投入水平。而对技术创新的产出水 平则以企业的专利数量为准, 包括企业首发上市当年已获 得和正在申请的发明专利数量。

\section{（3）控制变量}

控制变量主要包括企业所在地区, 企业规模、企业盈利 能力、企业偿债能力、企业知识密集程度等, 本文将其列 为控制变量。

表 I 研究变量解释与确定方法

\begin{tabular}{|c|c|c|c|c|}
\hline \multicolumn{2}{|r|}{ 变量类别 } & 变量计量 & 变量符号 & 变量值确定 \\
\hline \multirow{3}{*}{$\begin{array}{l}\text { 被 } \\
\text { 解 } \\
\text { 释 } \\
\text { 变 } \\
\text { 量 }\end{array}$} & 技术创新产出 & 专利数 & $\mathrm{P}$ & 企业上市当年获得的和正在申请的专利数量 \\
\hline & \multirow{2}{*}{ 技术创新投入 } & $\mathrm{R} \& \mathrm{D}$ 人员投入强度 & RDP & 技术人员/总员工人数 \\
\hline & & $\mathrm{R} \& \mathrm{D}$ 经费投入强度 & $\mathrm{RD}$ & 研究经费投入/企业营业收入 \\
\hline \multirow{4}{*}{$\begin{array}{l}\text { 解 } \\
\text { 释 } \\
\text { 变 } \\
\text { 量 }\end{array}$} & 有无风投 & & VCNO & 哑变量, 有风投持股为 1, 无为 0 \\
\hline & \multirow{3}{*}{ 风投持股比例 } & $0 \%-10 \%$ & \multirow{3}{*}{ VCI } & \multirow{3}{*}{ 前十大股东中风险投资机构持股比例之和 } \\
\hline & & $10 \%-20 \%$ & & \\
\hline & & $>20 \%$ & & \\
\hline \multirow{5}{*}{$\begin{array}{l}\text { 控 } \\
\text { 制 } \\
\text { 变 } \\
\text { 量 }\end{array}$} & 企业成长能力 & 净资产收益率 & ROE & 税后利润/所有者权益 \\
\hline & 偿债能力 & 资产负债率 & FL & 负债总和/资产总和 \\
\hline & 规模因素 & 总资产的对数 & LN (SIZE) & 资产总额取对数, 代表资产规模 \\
\hline & 知识密集程度 & 知识密集程度 & KI & 本科以上员工/总员工人数 \\
\hline & 地区因素 & 企业所在地区 & AERA & 亚变量, “北上广深”为 1 , 否为 0 \\
\hline
\end{tabular}

\section{B. 样本选取与数据来源}

本文以工信部等四部门联合发布的《中小企业划型标准 规定》 (2011) 为依据来界定中小企业。以在深圳证券交 易所创业板上市的公司作为中小企业样本来源。本文依据 以下要求选择样本企业：（1）2016 年 12 月 31 日前已在 创业版上市; （2）属于制造业企业; （3）企业首发上市 招股说明书上的从业人员在 $20-1000$ 人之间, 企业营业收 入在 300-40, 000 万元之间。通过篮选共选取 287 家公司 为本研究的样本。

本文对风险投资的界定标准遵循以下思路: 样本企业首 发上市时的招股说明书中, 若其前十大股东名称含有“创业 投资”、“风险投资”的，则将其定为有风险投资背景的企业， 没有则为无风险投资背景企业。此外，通过查阅中国风险 投资年鉴和清科研究中心的风险投资数据库中所录入的风 险投资公司名录, 若企业前十大股东中有名列其中的, 则 将该企业归为有风险投资背景的企业。本文的风险投资持 股比例为企业的前十大股东中风险投资机构持股比例之 和。根据以上规则, 287 家样本企业中有风险投资背景的 企业共 152 家，而无风险投资背景的企业共 135 家。
本文所使用的数据主要来源于巨潮资讯网 （http://www.cninfo.com.cn/）披露的创业板企业首发上市 招股说明书、国家知识产权局网站披露的公司专利数据, 少量数据来源于国泰安数据库、wind 数据库等并进行了必 要的整理。

\section{IV. 实证分析结果}

\section{A. 风险投资对中小企业技术创新资源投入的影响}

\section{1) 参与对投入的影响}

以研发经费投入强度 $(\mathrm{RD})$ 和研发人员投入强度 (RDP) 为被解释变量, 以是否有风险投资背景为解释变量, 构建 截面回归模型如下:

$R D=\beta_{0}+\beta_{1} V C N O+\beta_{2} A E R A+\beta_{3} R O E+\beta_{4} F L+\beta_{5} L N(S I Z E)+\beta_{6} K I$
$+\varepsilon$

$R D P=\beta_{0}+\beta_{1} V C N O+\beta_{2} A E R A+\beta_{3} R O E+\beta_{4} F L+\beta_{5} L N(S I Z E)+\beta_{6} K$

$I+\varepsilon$

模型（1）用于测度风险投资参与对企业研发经费投入 的影响, 而模型 (2) 则用于测度风险投资参与对创新人员 
投入的影响。运用统计软件 Eviews7.0 对 287 家样本企业进 行回归分析。结果如下。

表 II 模型（1）和模型（2）回归结果

\begin{tabular}{c|c|c}
\hline & 模型 (1) RD & 模型 (2) RDP \\
\hline \multirow{2}{*}{ VCNO } & $1.0938^{*}$ & $2.7805^{* *}$ \\
& $(1.803)$ & $(2.0532)$ \\
\hline \multirow{2}{*}{ AERA } & $1.5865^{* *}$ & $3.7046^{* * *}$ \\
& $(2.5331)$ & $(2.6498)$ \\
\hline \multirow{2}{*}{ ROE } & $-0.2452^{* * *}$ & -0.1217 \\
& $(-4.4039)$ & $(-0.9796)$ \\
\hline \multirow{2}{*}{ FL } & $-0.0696^{* * *}$ & -0.0166 \\
& $(-3.1904)$ & $(-0.3403)$ \\
\hline \multirow{2}{*}{ LN (SIZE) } & 0.7174 & -0.8532 \\
& $(1.2948)$ & $(-0.6898)$ \\
\hline \multirow{2}{*}{ KI } & $0.1742 * * *$ & $0.3778^{* * *}$ \\
& $(10.3086)$ & $(10.0143)$ \\
\hline R-squared & 0.3901 & 0.3223 \\
\hline Log likelihood & -867.8030 & -1098.269 \\
\hline F-statistic & 29.8534 & 22.1931 \\
\hline Prob(F- statistic) & 0.0000 & 0.0000 \\
\hline Obs & 287 & 287 \\
\hline
\end{tabular}

注: 括号内数值为 $\mathrm{t}$ 值, 括号外为估计系数, * *** ***分别表示在 $10 \% 、 5 \%$ 和 $1 \%$ 的显著水平 上显著。

从上表中的实证结果中, 可以得出以下结论:

模型 1 中, $\mathrm{F}$ 检验值为 29.8534 , 在 $1 \%$ 显著水平上显著, 说明模型总体显著通过 $\mathrm{F}$ 检验。从回归系数和 $\mathrm{t}$ 检验结果 可以看出 VCNO（风险投资参与）对企业技术创新研发经 费投入强度有正向影响, 并在 $10 \%$ 水平上通过了检验。 AERA 在 5\%水平上通过了检验, 系数符号为正, 说明地处 “北上广深”等经济发达地区的企业研发经费投入强度要明 显高于其他地区的企业。FL 在 $1 \%$ 水平上通过了检验, 系 数符号为负, 说明资产负债率低的企业, 研发经费投入力
度大于资产负债率高的企业。 $\mathrm{KI}$ 在 $1 \%$ 水平上通过了检验。 符号系数为正, 表明企业内知识水平越高, 企业内研发经 费投入力度越大。

(2)模型 2 中, $\mathrm{F}$ 检验值为 22.1931 , 在 $1 \%$ 显著水平上显 著, 说明模型总体显著, 通过 $\mathrm{F}$ 检验。由回归系数和 $\mathrm{t}$ 检 验结果可以看出 $\mathrm{VCNO}$ 对企业技术创新研发人员投入强度 有正向影响, 并 5\%水平上通过了检验。AERA 和 KI 分别 在 $1 \%$ 水平上通过检验, 系数符号为正, 说明二者能显著影 响企业技术创新研发人员投入强度。

上述实证结果表明，风险投资参与能够促进企业技术创 新资源投入, 而且研发人员投入力度比研发经费投入力度 更加显著。基于此，本文的假设 1“风险投资参与对企业的 技术创新投入有正向影响”通过检验。

\section{2) 持股比例对投入的影响}

在有风险投资背景的基础上，为进一步考察风险投资不 同持股比例对企业技术创新投入的影响，构建如下模型：

\section{$R D=\beta_{0}+\beta_{1} V C I+\beta_{2} A E R A+\beta_{3} R O E+\beta_{4} F L+\beta_{5} L N(S I Z E)+\beta_{6} K I+\varepsilon$}

$R D P=\beta_{0}+\beta_{1} V C I+\beta_{2} A E R A+\beta_{3} R O E+\beta_{4} F L+\beta_{5} L N(S I Z E)+\beta_{6} K I+$ $\varepsilon$

为说明不同持股比例对创新资源投入的影响, 本文将样 本企业中有风险投资的企业分为三个样本组。样本 1 , 全 部 152 家有风险投资背景的企业; 样本 2 , 全部 152 家中 风投比例在 $10 \%$ (含) 以下的企业; 共 78 家; 样本 3, 全 部 152 家中风投比例在 10\% (不含) 以上的企业, 共 74 家。 同样运用统计软件 Eviews7.0 对三个样本组中的企业的统 计指标进行回归分析, 结果如下。

表 III 模型(3)和(4)回归结果

\begin{tabular}{c|c|c|c|c|c|c}
\hline \multirow{2}{*}{} & \multicolumn{2}{|c|}{ 样本 1 } & \multicolumn{2}{c|}{ 样本 2 } & \multicolumn{2}{c}{ 样本 3 } \\
\cline { 2 - 7 } & RD & RDP & RD & RDP & RD & RDP \\
\hline \multirow{2}{*}{ VCI } & 0.0722 & -0.1179 & 0.2668 & -0.1374 & 0.0743 & -0.1218 \\
\cline { 2 - 7 } AERA & $(1.3704)$ & $(-1.0746)$ & $(1.2902)$ & $(-0.2266)$ & $(0.7236)$ & $(-0.6656)$ \\
\hline \multirow{2}{*}{ ROE } & $2.0228 * *$ & 2.7206 & $1.9448^{*}$ & 4.5654 & 2.4841 & 1.3184 \\
& $(2.0211)$ & $(1.3047)$ & $(1.9882)$ & $(1.5915)$ & $(1.3580)$ & $(0.4048)$ \\
\hline \multirow{2}{*}{ FL } & $-0.2688^{* * *}$ & -0.1617 & -0.1011 & -0.2281 & $-0.5067 * * *$ & -0.0709 \\
& $(-3.0052)$ & $(-0.8677)$ & $(-1.2368)$ & $(-0.9518)$ & $(-3.0186)$ & $(-0.2371)$ \\
\hline \multirow{2}{*}{ LN(SIZE) } & $-0.0985 * *$ & -0.0719 & $-0.0987 * * *$ & -0.0245 & $-0.1190 *$ & -0.1521 \\
& $(-2.5938)$ & $(-0.9084)$ & $(-2.2778)$ & $(-0.2312)$ & $(-1.7325)$ & $(-1.2431)$ \\
\hline \multirow{2}{*}{ KI } & $1.7023 * *$ & 0.0103 & 0.8820 & -1.4610 & $3.4121 *$ & 2.0708 \\
& $(1.9793)$ & $(0.0057)$ & 1.1668 & $(-0.6590)$ & $(1.9393)$ & $(0.6610)$ \\
\hline R-squared & $0.2143 * * *$ & $0.4433 * * *$ & $0.1700^{* * *}$ & $0.5598 * * *$ & $0.2567 * * *$ & $0.3189 * * *$ \\
\hline Log likelihood & $(8.0691)$ & $(8.0115)$ & $(6.7716)$ & $(7.6018)$ & $(5.4409)$ & $(3.7995)$ \\
\hline F-statistic & 0.4490 & 0.3727 & 0.5513 & 0.5148 & 0.4621 & 0.2557 \\
\hline Prob(F-statistic) & -479.9630 & -591.5335 & -213.1011 & -297.0213 & -247.5949 & -290.2825 \\
\hline Obs & 19.6913 & 14.3604 & 14.5363 & 12.5568 & 9.5949 & 3.8363 \\
\hline
\end{tabular}


实证结果显示, 样本 1 、样本 2 、样本 3 没有在任何显 著水平上通过检验, 由此得出以下结论: 风险投资持股比 例对企业技术创新研发人员投入强度和研发经费投入强度 没有显著影响。基于此，本文假设 3 “风险投资持股比例越 高, 被投资企业技术创新投入强度越大”未通过检验。但控 制变量中, 企业知识密集程度仍对技术创新投入存在显著 影响, 显著水平为 $1 \%$ 。

B. 风险投资对中小企业技术创新产出的影响

1) 参与对产出的影响
作为衡量技术创新产出的发明专利数是非连续型的非 负整数, 为计数变量 (Count Variable)。综合考虑其分布 特征, 本文决定选用负二项分布模型来考察风险投资参与 对技术创新产出的影响, 构建的模型如下:

$E(P / X)=\lambda=\exp \left(\beta_{0}+\beta_{1} V C N O+\beta_{2} A E R A+\beta_{3} L N(S I Z E)+\beta_{4} R O E+\right.$ $\left.\beta_{5} F L+\beta_{6} K I+\varepsilon\right)$

运用统计软件 Eviews7.0 对 287 家样本企业的风险投资 和技术创新等指标结合控制变量进行多次负二项回归分 析, 得到的实证结果如下:

表 IV 模型（5）回归结果

\begin{tabular}{|c|c|c|c|c|c|c|}
\hline & 1 & 2 & 3 & 4 & 5 & 6 \\
\hline VCNO & $\begin{array}{r}0.8132^{* * * *} \\
(7.4468)\end{array}$ & $\begin{array}{c}0.8254 * * * * \\
(7.5296)\end{array}$ & $\begin{array}{c}0.7994 * * * \\
(7.5285)\end{array}$ & $\begin{array}{c}0.7932^{* * * *} \\
(7.4115)\end{array}$ & $\begin{array}{c}0.7827 * * * * \\
(7.2499)\end{array}$ & $\begin{array}{c}0.7874 * * * * \\
(7.3172)\end{array}$ \\
\hline AERA & & $\begin{array}{c}0.1216 \\
(1.0750) \\
\end{array}$ & $\begin{array}{c}0.1121 \\
(1.0247) \\
\end{array}$ & $\begin{array}{r}0.1138 \\
(1.0404) \\
\end{array}$ & $\begin{array}{c}0.1075 \\
(0.9801) \\
\end{array}$ & $\begin{array}{c}0.0836 \\
(0.7601) \\
\end{array}$ \\
\hline LN(SIZE) & & & $\begin{array}{c}0.3989 * * * \\
(4.5471)\end{array}$ & $\begin{array}{c}0.4044 * * * \\
(4.5679)\end{array}$ & $\begin{array}{c}0.4337 * * * \\
(4.4259)\end{array}$ & $\begin{array}{c}0.4052 * * * \\
(4.0872)\end{array}$ \\
\hline ROE & & & & $\begin{array}{c}-0.0042 \\
(-0.4415)\end{array}$ & $\begin{array}{c}-0.0034 \\
(-0.3554)\end{array}$ & $\begin{array}{c}-0.0040 \\
(-0.4211)\end{array}$ \\
\hline FL & & & & & $\begin{array}{c}0.0027 \\
(-0.7013)\end{array}$ & $\begin{array}{c}0.0006 \\
(-0.1367)\end{array}$ \\
\hline KI & & & & & & $\begin{array}{c}0.0048 \\
(1.6320)\end{array}$ \\
\hline Adjusted R-squared & 0.1393 & 0.1340 & 0.1705 & 0.1724 & 0.1763 & 0.1903 \\
\hline Log likelihood & -1133.141 & -1132.559 & -1122.284 & -1122.187 & -1121.941 & -1120.589 \\
\hline Prob(LR-statistic) & 0.0000 & 0.0000 & 0.0000 & 0.0000 & 0.0000 & 0.0000 \\
\hline Obs & 287 & 287 & 287 & 287 & 287 & 287 \\
\hline
\end{tabular}

注: 括号内数值为 $\mathrm{t}$ 值, 括号外为估计系数, * ******分别表示在 $10 \% 、 5 \%$ 和 $1 \%$ 的显著水平上显著。

结果显示风险投资参与和企业的发明专利数呈正相关 关系, 并且六次回归都在 $1 \%$ 显著水平上显著, 有风险投资 背景的企业技术创新产出更多。这表明风险投资参与对企 业技术创新产出有促进作用，基于此，本文假设 2“风险投 资参与对企业的技术创新产出有正向影响”通过检验。

与此同时, 企业规模在 $1 \%$ 显著水平上显著, 说明企业 规模对技术创新产出有正向影响, 规模大的企业, 技术创 新产出更多。但实证结果显示, 企业注册地对技术创新产 出不具有显著影响。

\section{2) 持股比例对产出的影响}

在有风险投资背景的基础上, 为进一步考察风险投资不 同持股比例对企业技术创新产出的影响, 本文仍然采用负 二项分布回归模型，将风险投资持股比例代替模型（5）中 的 VCNO（有无风险投资），构建如下模型：

\section{$E(P / X)=\lambda=\exp \left(\beta_{0}+\beta_{1} V C I+\beta_{2} A E R A+\beta_{3} R O E+\beta_{4} F L+\beta_{5} L N(S I Z\right.$ $\left.E)+\beta_{6} K I+\varepsilon\right)$

本处也将有风险投资的 152 家样本企业分为三组, 同样 运用 Eviews7.0 对三个样本组中的企业的统计指标进行回 归分析, 结果如下。
表 V 模型（6）回归结果

\begin{tabular}{c|c|c|c}
\hline & 样本 1 & 样本 2 & 样本 3 \\
\hline \multirow{2}{*}{ VCI } & $0.0156^{* *}$ & $0.0832^{* *}$ & -0.0039 \\
& $(2.2214)$ & $(2.1706)$ & $(-0.4193)$ \\
\hline \multirow{2}{*}{ AERA } & -0.0503 & 0.1211 & $-0.3111^{* *}$ \\
& $(-0.4098)$ & $(0.6586)$ & $(-1.9617)$ \\
\hline \multirow{2}{*}{ ROE } & -0.0018 & -0.0072 & 0.0042 \\
& $(-0.1568)$ & $(-0.4648)$ & $(0.2698)$ \\
\hline \multirow{2}{*}{ FL } & -0.0065 & -0.0036 & $-0.0107^{*}$ \\
& $(-1.3107)$ & $(-0.4992)$ & $(-1.6992)$ \\
\hline \multirow{2}{*}{ LN(SIZE) } & $0.3670^{* * *}$ & $0.3474^{* *}$ & $0.3653 * *$ \\
& $(3.3237)$ & $(2.2715)$ & $(2.2920)$ \\
\hline \multirow{2}{*}{ KI } & 0.0008 & -0.0017 & 0.0024 \\
& $(0.2326)$ & $(-0.3415)$ & $(0.5529)$ \\
\hline R-squared & 0.0990 & 0.1369 & 0.1495 \\
\hline Log likelihood & -636.6051 & -313.3812 & -315.9766 \\
\hline Prob(LR-statistic) & 0.0000 & 0.0000 & 0.0000 \\
\hline Obs & 152 & 78 & 74 \\
\hline
\end{tabular}

注: 括号内数值为 $\mathrm{t}$ 值，括号外为估计系数，*、**、***分别表示在 $10 \% 、 5 \%$ 和 $1 \%$ 的显著水平

上显著。

结果显示样本 1 、样本 2 中 VCI 都在 $5 \%$ 显著水平上通 过检验, 系数符号为正, 而样本 3 中 VCI 对发明专利数没 
有在任何显著水平上通过检验, 并且系数符号为负。这种 结果表明, 有风险投资并且其持股比例在一定范围内（本 文是指在 $10 \%$ 以下），风险投资对企业技术创新产出有促 进作用。但随着持股比例的增加（本文是指超过 $10 \%$ ）, 其对企业技术创新的产出反而有不利影响。基于此, 本文 假设 4“风险投资持股比例越高, 被投资企业技术创新产出 水平越高”未通过检验。但企业规模这一控制变量, 所有样 本组在 5\%显著水平上都通过检验, 这可能表明公司规模越 大, 公司人才越多实力越强, 导致企业技术创新成果也越 多。

综上所述, 本文对研究假设的实证检验结果列表如下:

表 VI 研究假设检验结果

\begin{tabular}{lc}
\hline \multicolumn{1}{c}{ 研究假设 } & 验证结果 \\
\hline 假设 1: 风险投资参与对企业的技术创新 & 通过 \\
投入有正向影响。 & \\
假设 2: 风险投资参与对企业的技术创新 & 通过 \\
产出有正向影响。 & \\
假设 3: 风险投资持股比例越高, 被投资 & 未通过 \\
企业技术创新投入强度越大。 & \\
假设 4: 风险投资持股比例越高, 被投资 & 未通过 \\
企业技术创新产出水平越高 & \\
\hline
\end{tabular}

\section{V. 研究结论与讨论}

第一，风险投资参与对企业技术创新资源投入具有明显 的促进作用, 而且相较于研发经费, 对研发人员的投入促 进作用更大。这是风险投资作为一种权益性投资，在逐利 动机支配下, 动员了更多的企业资源用于能带来高额收益 的技术创新活动, 以增加自己的投资收益的结果。

第二, 风险投资在企业中持股比例越高并不意味着企业 创新资源投入就越多。这与风险投资最终要退出被投资企 业并意欲获得高额收益有关。风险投资者基于自利的目的, 当持股达到一定比例后, 基于退出的便利与退出时获得高 额收益的目的, 不再热衷于投资额大, 获利时间长的创新 活动, 而倾向于将企业资源配置到能迅速提高公司业绩的 项目中去, 以提高其退出时公司市值, 从而最大限度地获 取退出收益。

第三, 风险投资参与对企业技术创新产出具有显著的提 升作用。这往往与风险投资者的专业技能和丰富的社会化 网络资源有关。风险投资不仅为企业提供资金，还为企业 带来诸如营销、管理、市场等增值服务, 而“拥有丰富经验 的风险投资家对企业运营的高度参与可以提高企业在创业 和发展过程中各种项目的成功率”[7] (Keuschning, 2004）, 同时也帮助被投资企业提高创新绩效。

第四, 风险投资持股应控制在一定范围内才能对对企业 技术创新产出有促进作用。风险投资带来了企业所不具备 的资源, 可以提高企业创新能力从而导致企业创新产出的 增加。但随着持股比例的增大, 由于风险投资者短期收益 回报的诉求与创业团队长期收益诉求之间的矛盾, 将导致 企业创新产出的减少。
因此, 本研究建议, 为提高技术创新投入与产出水平, 中小企业的技术创新活动应该积极引进风险投资，但风险 投资的持股比例应控制在一定范围内。

\section{致谢}

本文是湖南省社会科学成果评审委员会 2018 年一般项 目《“五大发展理念”与企业社会责任关系研究》（项目编 号 XSP18YBC021）的阶段性成果之一。

\section{REFERENCES}

[1] Tykvova T.Venture capital in Germany and its impact on innovation[J].Social Science Research Network Working Paper,2010,15(7):86-88.

[2] XU Yu-lian, WANG Hong-qi.An empirical analysis of the role of China's financial development in technological innovation[J]. Statistics \& Decision,2011,27(21):144-146.

[3] WU Qiao-zhen.Path analysis of venture capital supporting independent innovation of high and new technology industry[J].Management World,2009,25(7):174-175.

[4] ZHAN Zheng-hua, TIAN Yang-yang, WANG Lei.The influence of syndicate venture capital on manufacturing enterprise's technology innovation capacity[J].Scientific Decision Making,2016, 23(10):47-61

[5] Ueda M,Masayuki Hirukawa.Venture Capital and Productivity[R].CEPR and University CEPR and University of Wisconsin at Madison Working Paper,2013.

[6] LU Wei. On the technological innovation principle of venture capital mechanism[J].Economic Research Journal,2002,48(2):48-56

[7] Keuschnig,Christian. Venture Capital Backed Growth[J].Journal of Economic Growth,2014,9(2).

[8] LIU Liang.Research on the impact of venture capital on technological innovation of high and new technology enterprises in China-Empirical research based on experience data of China's GEM high and new technology enterprises[J].Market Weekly,2013,36(3):145-147.

[9] Schwienbacher.Venture Captial:Project Evaluation,Screening and Expropriation[J].Journal of Finance, 2012,59(2).

[10] XU Xin, XIA Yun. Characteristics of venture capital, venture capital exit through IPO and firm performance: an empirical research on listed companies of ChiNext Market[J].Business Management Journal,2015,37(5):97-107.

[11] XIE Ya-ping, SONG Chao-li.The impact of venture capital on technological innovation of enterprises[J].Studies in Dialectics of Nature,2016, 32 (7) :57-61.

[12] WANG Yu-rong, LI Jun.Empirical study of the effect of the venture capital on the independentinnovation of SMEs: Based on the data from SME board [J].Journal of Shandong University of Science and Technology(Social Sciences), 2009, 11(1):47-52

[13] ZHAO Wu,LI Xiao-hua. ZHU Ming-xuan, PANG Jia-lan. Study on Venture capital, R\&D investment in technological innovation outputs differentiated impact[J]. Science and Technology Management Research,2015, 35(7):1-11.

[14] Bottazzietal,Gatti S,Perrini F.Are Venture Capitalists a Catalyst for Innovation[J].European Financial Management,2012,15(1). 